\title{
Should the Therapeutic Apheresis Method Be Added to Treatment in the COVID-19 Outbreak?
}

\author{
Mustafa Şahin $^{\mathrm{a}} \quad$ Abdülkerim Yıldız $^{\mathrm{b}}$ \\ ${ }^{a}$ Department of Medical Biochemistry, Hitit University Erol Olçok Training and Research Hospital, Çorum, Turkey; \\ ${ }^{b}$ Department of Hematology, Hitit University Erol Olçok Training and Research Hospital, Çorum, Turkey
}

Dear Editor,

The coronavirus outbreak, which started in Wuhan, a city in China, in December 2019, soon spread across the world and was declared a pandemic by the World Health Organization. The disease was identified as novel coronavirus-induced (SARS-CoV-2) COVID-19. No effective antiviral agent or vaccine has been produced for the disease, and deficiencies of medical protective equipment and respiratory support devices are causing difficulties in the fight against COVID-19.

In this letter, we would like to bring into sharp relief a technique used in the treatment of certain viral diseases and present it to scientific argumentation. We present the potential role of therapeutic apheresis for the purpose of viral eradication or reducing viral load, which may be used in the treatment of COVID-19.

In this therapeutic method, the plasma separated from the patient's blood is passed through a filter suitable for the virus to be removed using a medical device, thus reducing the circulating viral load. Then, the cleaned plasma free of the virus is returned to circulation.

In particular, increased drug treatment effectiveness with reduced viral load in hepatitis B and C and HIV patients has been shown in many studies that are scientifically clear. The relationship between decreased viral load and survival and control of disease is linear $[1,2]$. There are no enough data about the effect of serum viral load on the pathogenesis of COVID-19.

karger@karger.com

(c) 2021 S. Karger AG, Basel

www.karger.com/bpu

Karger!
In Chile, Vial et al. [3] observed that plasmapheresis treatment decreased the case fatality rate of hantavirus cardiopulmonary syndrome caused by Andes virus. In 2015, Liu et al. [4] reported that plasma exchange and continuous venovenous hemofiltration (different apheresis techniques) might be useful for the treatment of severe avian influenza A (H7N9). In 2018, Koch et al. [5] reported that lectin affinity plasmapheresis eliminated Middle East respiratory syndrome coronavirus and Marburg virus in vitro. They suggested that considering the high lethality and lack of established treatment options, lectin affinity plasmapheresis should be evaluated in vivo [5]. When considering the genetic similarity of MERS$\mathrm{CoV}$ and the novel coronavirus, the plasmapheresis technique, which is effective in the treatment of MERS-CoV, may be used in COVID-19 patients.

The pathogenesis of COVID-19 is still unclear; however, recent studies have demonstrated that the immune response is crucial for controlling and removing COVID-19. Especially in critically ill patients, elevated levels of proinflammatory cytokines and a consequent cytokine storm may contribute to severe respiratory disease. Therapeutic plasma exchange (TPE) can be considered to be an effective method to solve this problem, with the belief that it removes cytokines from the blood. We have no case experience, but there are some data in the literature that suggest TPE for COVID-19 patients. Adeli et al. [6] reported a case series of 8 patients with COVID-19 who 
were in the septic shock stage and had ARDS. They used corticosteroid therapy and interferon administration in combination with TPE. Patients' respiratory status improved significantly after plasmapheresis, and they concluded that TPE suppressed the cytokine storm and reduced the inflammatory status and helped the patients to defeat COVID-19 [6]. Another case, reported by Lin et al. [7], is of a 52-year-old woman who was diagnosed with COVID-19 infection and had endotracheal intubation because of progressive pneumonia. They performed TPE and continuous venovenous hemofiltration alternatively. They observed improvement in clinical manifestations and radiographic images and laboratory investigations and suggested that it confirms the suspicion of cytokine storm as the cause of those problems [7]. Experiences from past infectious cases with other viruses and these COVID patients may demonstrate the major advantage of TPE comes from the exchange between plasma of the patient and protective factors from fresh frozen plasma that had been depleted by the infection. However, there is a doubt that the exchange of plasma might also replace consumed protective factors that are critical to maintain microcirculatory flow. With regard to COVID-19, it has been recognized that humoral immunity is of critical importance in clearing SARS-CoV-2, and treatment with convalescent plasma containing virus-specific neutralizing antibodies has been suggested as a potential treatment in critically ill patients. In this context, some authors suggested to be very cautious in recommending TPE using plasma from nonspecific donors because this procedure itself might remove critically important neutralizing antibodies against SARS-CoV-2. Stahl et al. [8] detected SARS-CoV-2-specific IgG and IgA antibodies in the waste bag plasma. However, they suggested that TPE with plasma collected from reconvalescent donors that carry specific neutralizing antibodies might be both effective and safe [8].

In conclusion, it is challenging to clarify the role of TPE in treatment of COVID patients since those experiences in various studies were heterogeneous. Besides, some concerns about impact on cytokine and antibody levels may raise doubts as to whether it will benefit. By calculating profit and loss for patient treatment, the method of apheresis for therapeutic purposes might be presented for scientific argumentation. The age-group in which the method can be applied, the clinical status of the patients, severity of the disease, and the presence of comorbidity should be consulted by different medical disciplines. Prospective, randomized, controlled trials are needed to justify the role of this treatment modality.

\section{Conflict of Interest Statement}

The authors have no conflicts of interest to declare.

\section{Funding Sources}

The authors did not receive any funding.

\section{Author Contributions}

M.Ş.: conceptualization, methodology, writing - original draft, writing - data curation, review, editing, and supervision. A.Y.: writing - original draft, review, editing, and supervision.

\section{References}

1 Schettler V, Monazahian M, Wieland E, Ramadori G, Grunewald RW, Thomssen R, et al. Reduction of hepatitis $\mathrm{C}$ virus load by H.E.L.P.-LDL apheresis. Eur J Clin Invest. 2001;31(2):154-5.

2 Mednikov RV, Rabinovich VI, Kizlo SN, Belyakov NA, Sokolov AA. Double filtration plasmapheresis in treatment of patients with co-infection of hepatitis $C$ and human immunodeficiency virus. Ther Apher Dial. 2016; 20(4):413-9.

3 Vial PA, Valdivieso F, Calvo M, Rioseco ML, Riquelme R, Araneda A, et al. Hantavirus Study Group in Chile. A non-randomized multicentre trial of human immune plasma for treatment of hantavirus cardiopulmonary syndrome caused by Andes virus. Antivir Ther. 2015;20(4):377-86.
4 Liu X, Zhang Y, Xu X, Du W, Su K, Zhu C, et al. Evaluation of plasma exchange and continuous veno-venous hemofiltration for the treatment of severe avian influenza A (H7N9): a cohort study. Ther Apher Dial. 2015;19(2): $178-84$.

5 Koch B, Schult-Dietrich P, Büttner S, Dilmaghani B, Lohmann D, Baer PC, et al. Lectin affinity plasmapheresis for middle east respiratory syndrome-coronavirus and Marburg virus glycoprotein elimination. Blood Purif. 2018;46(2):126-33.
6 Adeli SH, Asghari A, Tabarraii R, Shajari R, Afshari S, Kalhor N, et al. Therapeutic plasma exchange as a rescue therapy in patients with coronavirus disease 2019: a case series. Pol Arch Intern Med. 2020;130(5):455-8.

7 Lin JH, Chen YC, Lu CL, Hsu YN, Wang WJ. Application of plasma exchange in association with higher dose CVVH in cytokine storm complicating COVID-19. J Formos Med Assoc. 2020;119(6):1116-8.

8 Stahl K, Bode C, David S. First do no harmbeware the risk of therapeutic plasma exchange in severe COVID-19. Crit Care. 2020; 24(1):363. Published 2020 Jun 18. 